\title{
Photovoltaic solar farm: earthing system design for cost reduction and system compliance
}

\author{
Mohamad Nassereddine ${ }^{1}$, Khaled Ali $^{2}$, Chadi Nohra ${ }^{3}$ \\ ${ }^{1,2}$ Department of Electrical and Computer Engineering, American University in Dubai, United Arab Emirates \\ ${ }^{3}$ Department of Electrical and Computer Engineering, Beirut Arab University, Lebanon
}

\begin{tabular}{l} 
Article Info \\
\hline Article history: \\
Received Jun 26, 2019 \\
Revised Dec 10, 2019 \\
Accepted Dec 16, 2019 \\
\hline
\end{tabular}

Keywords:

Earth potential rise

Fault current distribution

PV grounding

Split factor

\begin{abstract}
The increasing demand for green sustainable energy source led to a worldwide increase in the installation of large scale photovoltaic (PV) farms. To ensure the PV farms compliance with safety and operational guidelines, earthing systems are essential component of the design. An adequate earthing system for low, medium, and high voltage areas of the PV farm ensures energy under fault condition and system malfunction are absorbed without exceeding the desirable limits. The work in this paper captures the soil resistivity and the earthing system requirements for each voltage level. The paper highlights the boundaries for combined or separate earthing system between low, medium and high voltage. Furthermore, the works focus on the system cost and maintenance without jeopardizing system integrities and safety compliance. Case study is also included.
\end{abstract}

Copyright () 2020 Institute of Advanced Engineering and Science. All rights reserved.

\section{Corresponding Author:}

Chadi Nohra,

Department of Electrical and Computer Engineering,

Beirut Arab University, Tripoli Lebanon, United Arab Emirates.

Email: c.nohra@bau.edu.lb

\section{INTRODUCTION}

Electrical power form an indispensable part of human comfort and economic developments. An electric power outage can cause major disturbance to human daily activity, and might burden the global economy. Moreover, generation of electric power is heavily dependent on fossil fuel. The Co2 emissions from this generation process negatively affects the environment. In addition to the environmental impacts, the depletion of fossil fuel puts additional burden on government and researchers to find a green sustainable source for electrical power generation.

Solar energy is one of the green sustainable sources that can be used as an alternative to fossil fuels. Photovoltaic (PV) technologies covert sun radiation into direct current (DC) energy, without the negative impact associated with utilizing fossil fuel. The United Nations, supported by governments, encourage the deployments of PV system for small, medium and large-scale projects. Large scale projects are formed with large number of PV panels across large area, as shown in Figure 1 [1]. The majority of electrical networks operate on alternating current (AC) system. Thus, in addition to generating DC from sun radiation, the PV farm converts DC to AC, for network deployments. For a large PV farm, after obtaining the AC power, the system will step up the voltage using two stages:

- Low voltage to medium voltage $(11 \mathrm{kV}$ to $133 \mathrm{kV})$

- Medium voltage to high voltage (66kV and up)

The high voltage side is connected to a transmission line network to transmit the generated power to the load. To ensure the system is complying with safety regulations and operational guidelines, earthing system (also known as grounding system) is required throughout the plant. To comply with standards and policies, the earthing system is required for the DC, low, medium and high voltage areas. The earthing system is composed of the earth grid, the earth cable, and the cable screen (or the overhead earth wire) [2-7]. 
The earth grid is formed by steel foundations, electrodes and mesh. The earth grid is located at the PV panels, as well as, at the low, medium and high voltage substations. PV farms are exposed to DC and AC fault conditions. The role of the earth grid is to absorb the fault energy, which prevents human injury or damage to the equipment.

Currently, IEEE standards [2, 5] are the main references for earthing design procedures. These standards are based on substations and has no reference or special procedures for large PV farm earthing design. Following the current IEEE standards could lead to an unnecessarily complex system, which has impact on cost and environment. The main contribution of this paper is a step-by-step guide to design the earthing system for large scale PV farms. The paper focuses on system compliance while reducing project costs, maintenance requirements without jeopardizing the safety aspect of the design. Another contribution of this paper is analysis of the impact of various soil resistivity structure on the earthing design of PV farm. The provided analysis includes DC, low, medium, and high voltage areas. This analysis should provide the designer with the required initial assessments. Finally, this paper considers the various voltage areas, i.e. DC, low, medium, and high voltages, in the earthing design process.

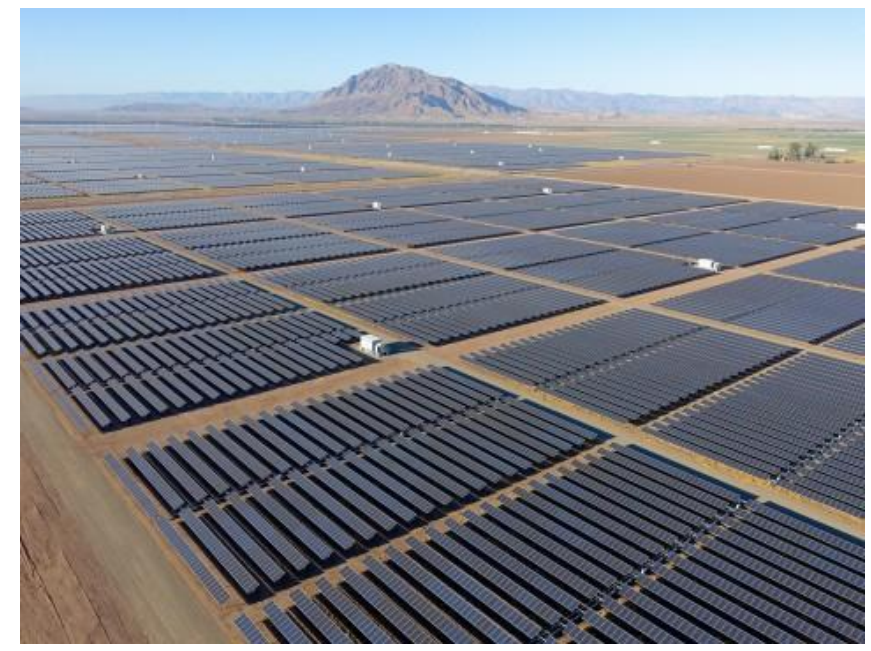

Figure 1. Large scale PV farm

\section{THEORETICAL STUDY}

\subsection{Photovoltaic farm design}

The design and layout of PV farms is discussed in this section. The power of the PV panels varies between 100 to 370 watts. For large PV farm, the required number of PV panels $\mathrm{N}_{\mathrm{PV}}$ is determined by (1):

$$
N_{P V}=\frac{P_{F}}{P_{P V}}
$$

where $P_{F}$ is the PV farm power capacity in Watts and $P_{P V}$ is the individual PV panel power in Watts. The PV panels are installed in strings and arrays to suit the installed inverter rating capacity. The number of PV panels per inverter is computed using (2).

$$
N_{P V-i}=\frac{P_{i}}{P_{P V}} \times M
$$

where $P_{i}$ is the chosen inverter maximum power point (MPPT) rated power as per the manufacturer in Watts, $P_{P V}$ is the individual PV panel power in Watts, and $M$ is the number of MPPT at the chosen inverter. Figure 2 shows a layout of PV farm. As shown in Figure 2, the AC side of the inverter is fed to a step-up transformer. Also, the high side of the transformer is fed to the high voltage substation for another voltage step-up. Cables with screens are used to connect the medium transformers to the high voltage substations. 


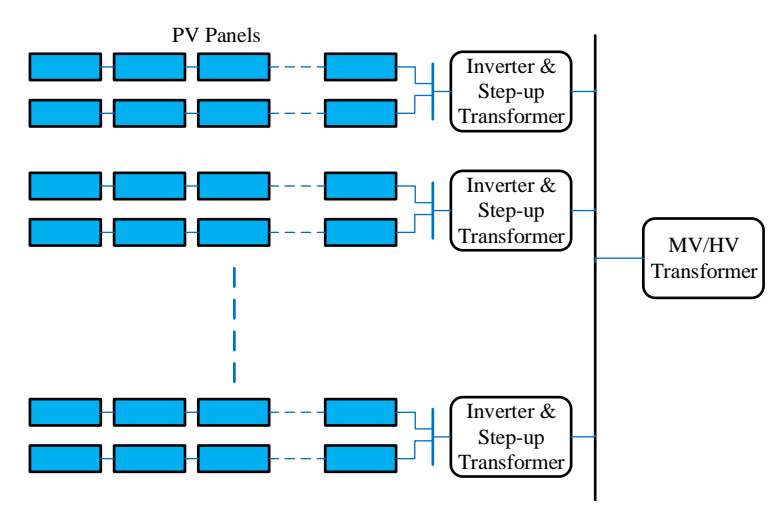

Figure 2. PV farm layout showing the panels, inverter and transformer

\subsection{Soil resistivity}

The earth grid resistance of the grounding system is governed by the size of the conductive materials embedded into the ground, and the soil resistivity $(\rho)$ of the area. Therefore, soil resistivity forms an important element when it comes to grounding system. Measuring soil resistivity is required to determine the soil's ability to retard the conduction of an electric current. Soil resistivity values typically range from about 2 to 10,000 $\Omega-\mathrm{m}$, but more extreme values are not unusual. Table 1 shows the soil resistivity range for different soil types. The soil resistivity value significantly impacts the grid resistance. The grid resistance in turn reflects on the earth potential rises and voltage transfer. Therefore, a more accurate soil resistivity value is required for compliant design. Any of the soil resistivity methods, such as Wenner method, the Schlumberger array, or the driven rod method can be used to determine the soil resistivity [1-11].

Table 1. Typical soil resistivity of various type of soil

\begin{tabular}{lc}
\hline \multicolumn{1}{c}{ Type of Soil or water } & $\rho(\Omega-\mathrm{m})$ \\
\hline Sea Water & 2 \\
Clay & 40 \\
sGround well and spring water & 50 \\
Clay and Sand mix & 100 \\
Shale, Slates, Sandstone & 120 \\
Peat, Loam and Mud & 150 \\
Lake and Brook Water & 250 \\
Sand & 2000 \\
Morane Gravel & 3000 \\
Ridge Gravel & 15000 \\
Solid granite & 25000 \\
Ice & 100000 \\
\hline
\end{tabular}

\subsection{Grid resistance}

The earth grid resistance of a single electrode $R_{g}$ is calculated by (3) [2-14]

$$
R_{g}=\frac{\rho}{2 \pi L}\left(\ln \left(\frac{8 L}{d}\right)-1\right)
$$

where $\rho$ is the soil resistivity, $L$ is the buried length of the electrode in meters, and $d$ the diameter of the electrode in meters. Figure 3 shows the resistance of $0.5 \mathrm{~m}$ electrode, with a cross-sectional area of 70 square millimeters, under various soil resistivity conditions. Figure 3 clearly reflects the increase in earth grid resistance value of a single electrode with the increase in the soil resistivity.

The PV panels are installed on a support structure, which has concrete foundations. These foundations have conductive steel materials which makes it suitable to act as an electrode. It is worth noting, that the PV system cannot exists without the supporting structure, therefore these structures form an indispensable element of the PV farm. Figure 4 shows the panel installations in PV farm. The large number of PV panels reflect large number of steel foundations. Combining these steel foundations has the potential to form low grid resistance, pending soil resistivity values. During the assessment within this paper, each leg is considered as an electrode. 


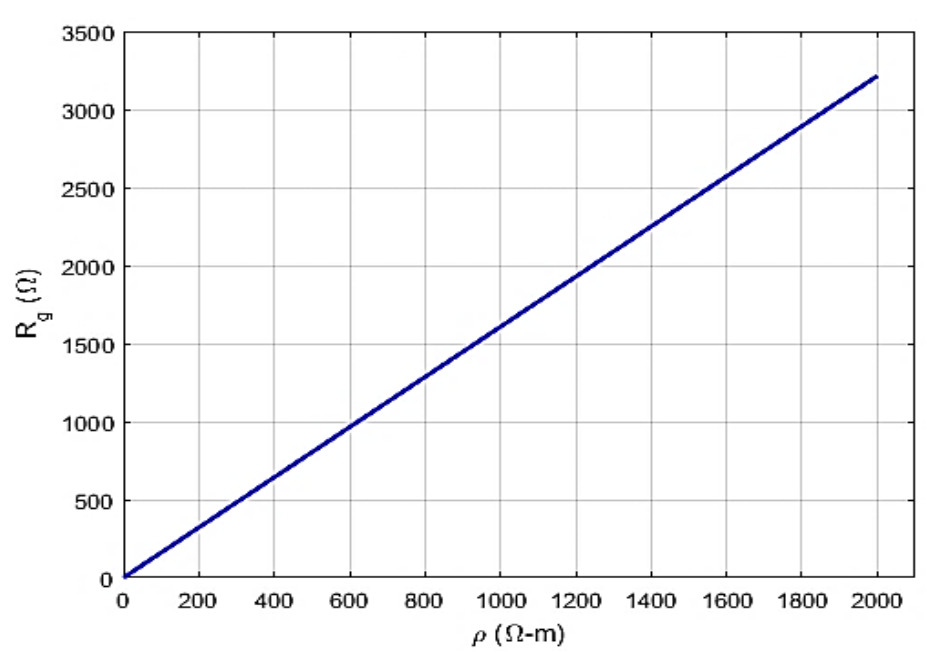

Figure 3. Single electrode grid resistance for varoious soil resistivity

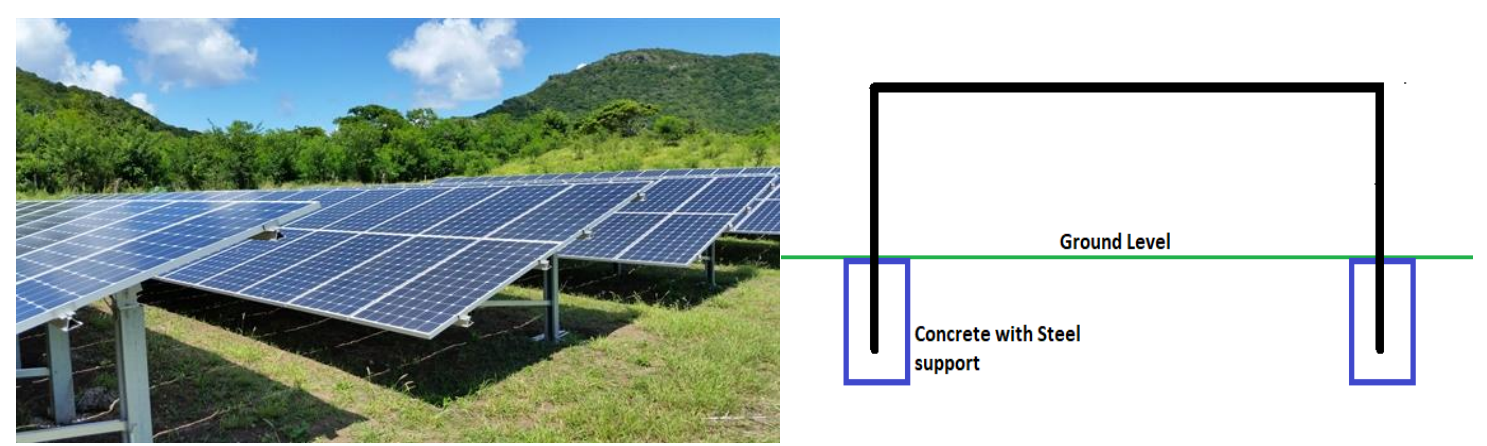

Figure 4. PV panels installation in large farm and its foundation embedded into the ground [11]

For a row of PV panels, each foundations is connected to the next one using the steel of the structure that support the panels. Let $R_{s}$ denote the resistance of the steel structure between two foundation poles, $\mathrm{R}_{\mathrm{a}}$ denote the resistance of the steel structure above ground, and $R_{f t}$ denote the footing resistance of the foundation pole. Figure 5 shows the resistance circuit of a row of PV panels. Note that $R_{f t=} R_{g+} R_{a}$, where $\mathrm{Rg}$ is obtained from (3) represents the resistance below ground for the foundations of the supporting structure. Since $\mathrm{R}_{\mathrm{g}}>>\mathrm{R}_{\mathrm{a}}$, therefore $\mathrm{R}_{\mathrm{ft}} \approx \mathrm{R}_{\mathrm{g}}$.

For a long row where it is considered an infinite system, analysing the circuit in Figure 6 to determine the total resistance of the row. " $R_{\text {inf }}$ " is the infinite resistance as seen from the end of the row. Infinite system definition: when the input resistance at the edge of the row cannot capture the entire length of the system, therefore only a section of the row is considered for the input resistance.

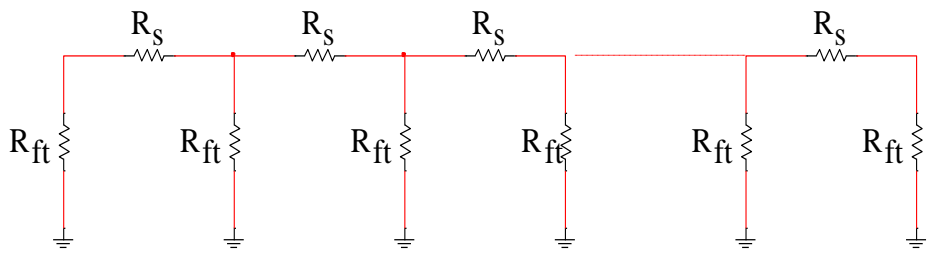

Figure 5. Resistance circuit for the row

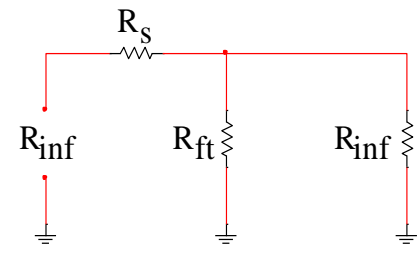

Figure 6. circuit analysis for the long row 
Analyzing the circuit in Figure 6 generates in (4) [2, 5]. Figure 7(a) illustrates the infinite input resistance of a long row using footing resistance from Figure 5. For the simulation, $\mathrm{R}_{\mathrm{s}}$ is assumed to be $0.1 \Omega$.

$$
R_{\text {inf }}=\frac{R_{s}}{2}+\sqrt{R_{S} R_{f t}}
$$

Figure 7(b) shows the input impedance for an infinite row with different foundations details. The figures show the foundation depth changes to represents different electrode characteristics. It is clearly shown from the figure that the foundation details play an important role along with the soil resistivity to determine the infinite input resistance value. Based on the above information, it is important to determine the length for the row to be considered an infinite system. The paper uses (5) to determine the infinite length $L_{\text {inf }}[15,16]$.

$$
L_{i n f} \sqrt{\frac{R_{S}}{R_{f t}}}>2
$$

Figure 8 shows the length of the row to be considered as an infinite system for different foundations characteristics. Based on the simulated data, it is clearly shown that in solar farms, infinite conditions are applicable as the row usually are formed by 100 s of panels which equate to $100 \mathrm{~s}$ of meters. The resistance as per (4) is used as an input resistance at the inverter transformer earth grid.

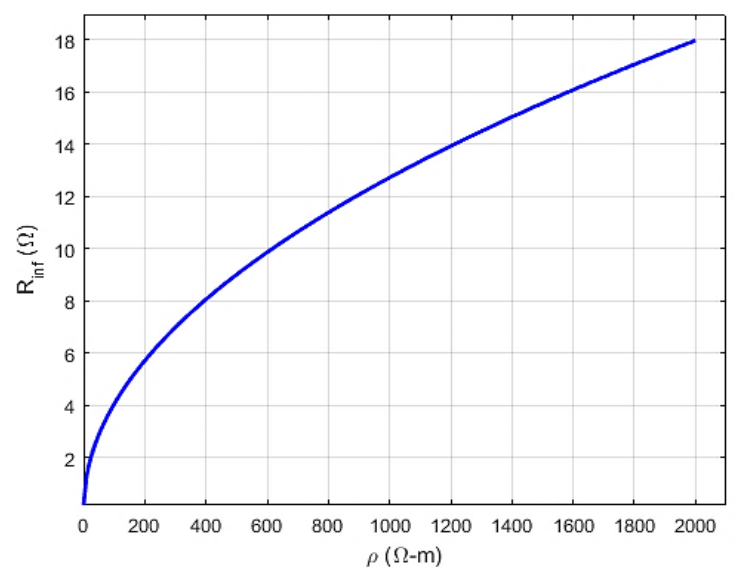

(a)

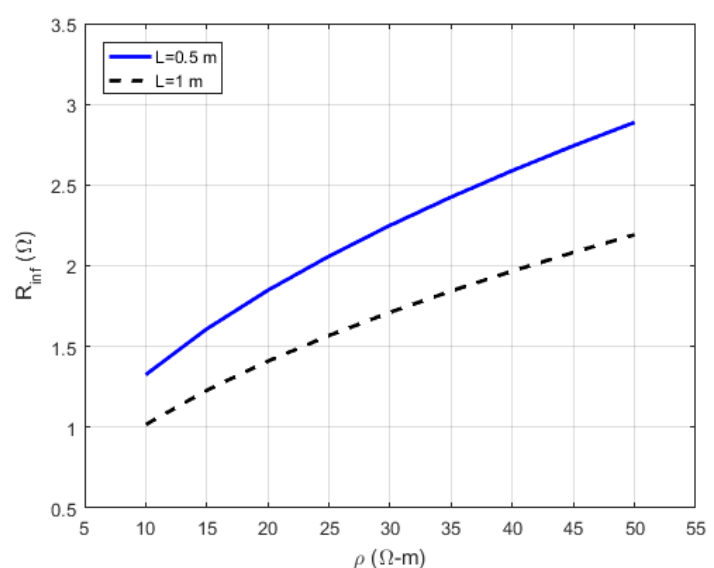

(b)

Figure 7. (a) Infinite input resistance for a long infinite row, (b) Infinite input resistance for an infinite row under different foundation

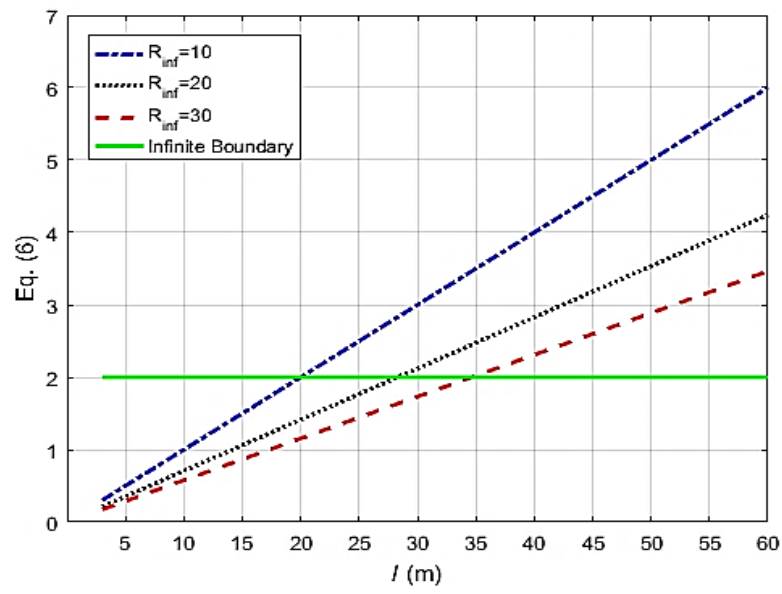

Figure 8. Infinite length for row under different foundation details 


\subsection{Earth grid layout}

Following the layout in Figure 2, the earth grid of each section can be bonded using the cable screen or cable trays to the remaining earth grids. Figure 9(a) shows the proposed circuit for the combined earthing system of the PV farm. Combining the earth grid offers numerous branches for the fault current distributions, in addition to voltage transfer between these grids. Depending on the fault current magnitude and earth grid resistance, the voltage rise due to current distribution could reach unsafe limit and cause damage to apparatuses and risk to humans. Pending the transformer arrangement (Delta-Wye) and if it is grounded, the ground fault current condition will be determined. The ground fault at the HV substation can be fed from the utility and the entire PV farm. This condition could increase the ground fault current due to utility fault conditions. Under fault condition, the current split is governed by two laws:

- Current divider rule

- $\quad$ Mutual coupling between faulted phase and return path

Figure 9(b) represents a fault condition, the current into the combined earth grid is governed by current divider rule and the mutual coupling between the faulted phase and cable screen. The fault current distribution between the earth grids (HV, MV and PV earth grid) is governed by the current divider rule without mutual coupling. The screen current can be computed using (6) [16]

$$
I_{g}=I_{f}\left(\frac{Z_{S c r-i n}-Z_{m}}{Z_{S c r-i n}+R_{g}}\right)
$$

where $I_{g}$ is the total grid fault current (for the combined grids), $I_{f}$ is the fault current, $Z_{s c r-i n}$ is the cable screen input impedance, $Z_{m}$ is the mutual coupling between the faulted phase and the cable screen, and $R_{g}$ is the combined earth grids (HV, MV and PV earth grids). After determining $I_{g}$ using in (6), current divider rule is applied to split $I_{g}$ into the different earth grids (HV earth grid, MV earth grid and PV earth grid).

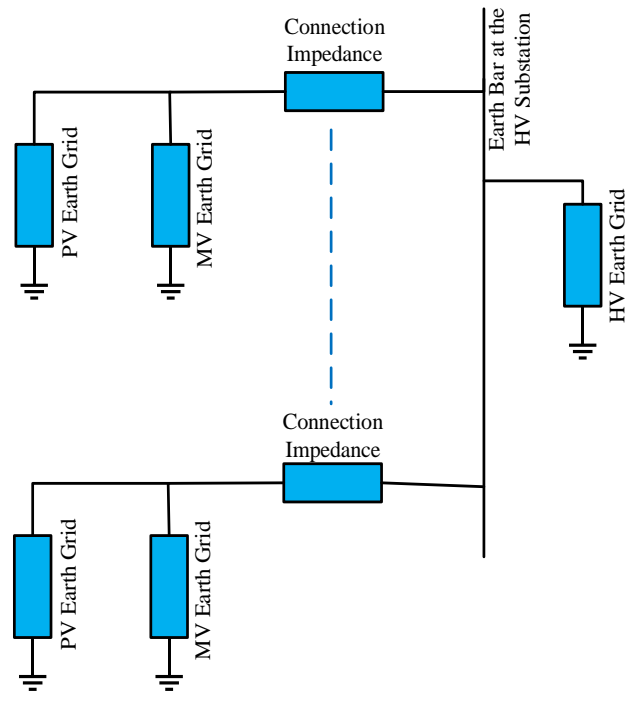

(a)

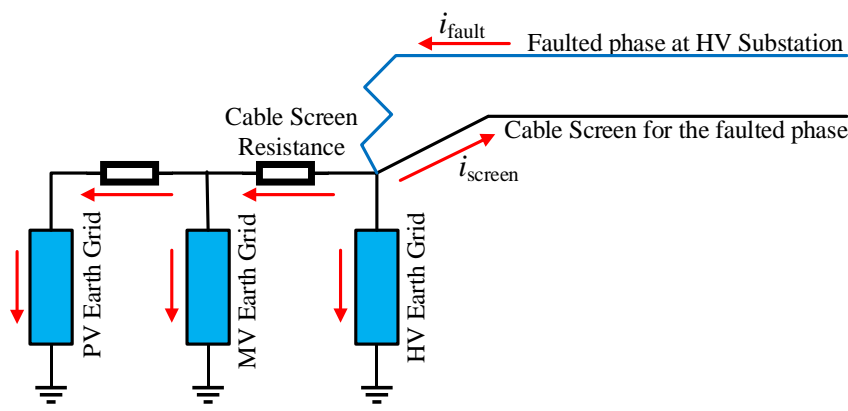

(b)

Figure 9. (a) Earth grid circuit layout; (b) fault condition with mutual coupling

\section{PROPOSED DESIGN DIAGRAM}

One of the paper's main contributions is presented in this section. As highlighted within the introduction, the current standards and procedures do not reflect the optimum process for large PV farm earthing deign. The paper utilized the available information within the current standards [2-7], and the available research outputs [13-20], to determine the optimum steps to ensure the earthing design complies with safety and operation policies. Note that this is done while reducing implementation cost and environmental impact of the project. Based on the theoretical section analysis, the paper proposes the design diagram shown in Figure 10. 


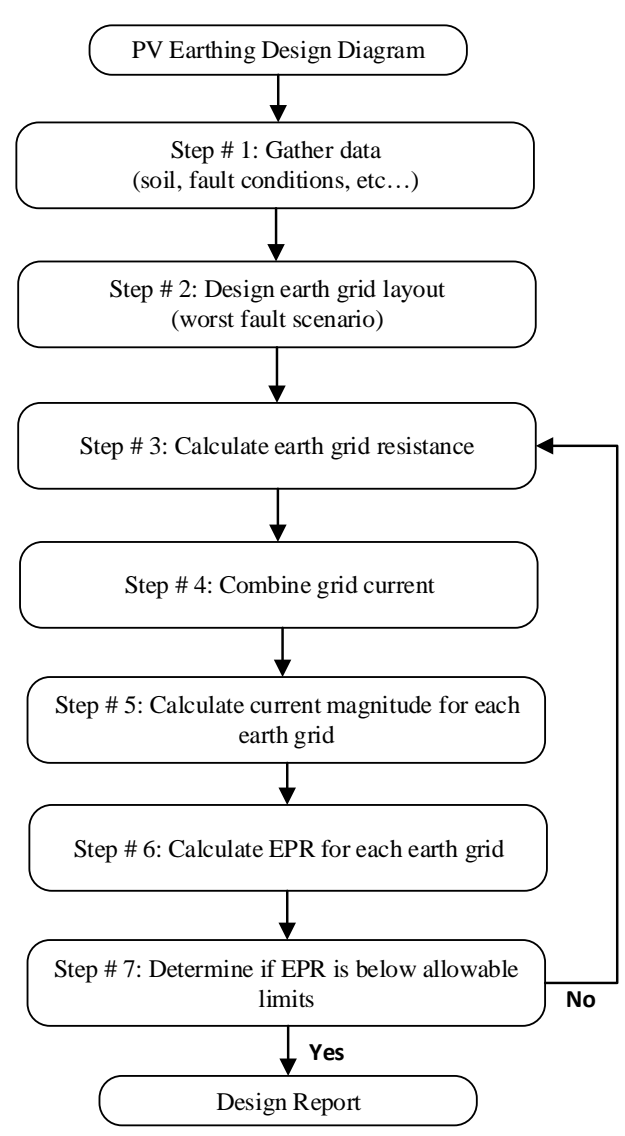

Figure 10. Proposed design diagram for PV farm earthing system

The aim of the diagram is to set the optimum steps for the design when it comes to PV solar farm earthing system. The diagram aid the designer to determine the required steps to ensure the system is compliant to safety and operational requirements. From the diagram, step \#3 form the area where the earth grid resistance is computed based on the proposed system. Based on the results from step \#7, the earth grid design will be altered to ensure the earth potential rise (EPR) is within the allowable safety limits. For allowable safety limits, IEEE 80 and references [5] can be used. The theoretical section highlights that the foundation of the PV structure can be used as electrodes to reduce the earth grid of the PV farm. The combined footings resistance can be computed using (4) along with the maximum length as per (5). These concepts is the same as transmission line input resistance, which is verified in the following references [20-25]. These equations aid the designer to complete the assessment using excel or Matlab engineering software.

The proposed diagram set the steps required to complete the adequate design. The diagram ensures the cost of the earthing system is controlled by ensuring only the required electrode is installed. Additional electrodes are installed to suit steps $2 \& 3$. By starting the design assessment using the foundations as electrodes and then complete the split factor calculations, the designer has the opportunity to control the cost of the earthing system by reducing the number of electrode required without consideration of the structure foundations. The cost reductions add great value to the engineered earthing system and has positive impact on the project overall budget. In this section, it is explained the results of research and at the same time is given the comprehensive discussion. Results can be presented in figures, graphs, tables and others that make the reader understand easily $[2,5]$. The discussion can be made in several sub-chapters

\section{THEORETICAL DISCUSSION}

Safety is always the number one priority during any phase of a project. Earthing system is critical because, in addition to maintaining adequate operation of the PV farm, it ensures safety of the PV farm. Currently, one of the major issues related to designing earthing system for large PV farms is the absence of clear set of procedures that govern the designer decisions. For example, for conventional high voltage 
substations and transmission lines, IEEE and other standards set clear design steps to ensure system compliance while taking into consideration the cost and maintenance requirements. However, a unified design steps for large PV farms does not exist. To resolve this major issue, this paper introduces a design diagram, shown in Figure 10. This design diagram, which adheres to the various standards and publications, can be used as a guideline for the minimum requirements throughout the design process. The proposed design diagram also introduces the first earthing design diagram for large PV farms. Note that this design diagram can be used as a basis for earthing system design for alternative renewable energy projects such as wind farms.

\section{CASE STUDY}

A 200MW PV solar farm is planned for Lebanon Bekaa valley, the PV panels are installed in the following arrangement:

- 113 rows of panels, 5000 panels per row

- Footing separation is $4 \mathrm{~m}$, footing depth is $1 \mathrm{~m}$, and footing rod cross-sectional area is $120 \mathrm{~mm} 2$

- The steel structure is made of 90x90 square galvanized steel with thickness of $7 \mathrm{~mm}$

- $\quad$ Resistivity of steel structure $13.8 \times 10^{\wedge}(-8) \Omega-\mathrm{m}$ and Soil resistivity of the area is $100 \Omega-\mathrm{m}$

- $\quad$ Every row has its own step up transformer to $132 \mathrm{kV}$

- The step-up transformer grounding is connected to $132 / 220 \mathrm{kV}$ grounding using $120 \mathrm{~mm} 2 \mathrm{screen}$.

- $\quad$ The $220 \mathrm{k} \mathrm{V}$ feeder has a 48 core OPGW as the OHEW.

- The separation between the OHEW and the lowest phase is $4.1 \mathrm{~m}$

- The highest fault current supplied by the $220 \mathrm{kV}$ is $15 \mathrm{kA}$ with $150 \mathrm{~ms}$ clearance time

- $\quad$ The $220-\mathrm{kV}$ substation earth grid is $0.89 \Omega$

The substation earth grid current is calculated using the split factor and the guidelines as per the IEEE 80 standard [5]. The grid current is computed to be $2906 \mathrm{~A}$ and the total EPR at the $220 \mathrm{kV}$ substation is $2.586 \mathrm{~V}$. Note that the EPR is very high and the design must reduce the $220 \mathrm{kV}$ earth grid resistance to reduce the EPR to acceptable limit. By following the proposed design diagram, the design is completed using the same inputs. The design commences by calculating the resistance of a single foundation of the steel structure that supports the PV panels via (1). Thus, for soil resistivity $\rho=100 \Omega-\mathrm{m}$, the footing resistance $\mathrm{Rft}=\mathrm{Rg}=87.1 \Omega$. The connection resistance between the two foundations $\mathrm{Rs}$ is calculate to be $0.4558 \mathrm{~m} \Omega$. From (5) and the calculated values of Rft and Rs, for each row to be considered as an infinite system Linf $>874.26 \mathrm{~m}$, since each row is composed of 5000 panels, i.e. the length of each row is $5 \mathrm{~km}$, the infinite system condition is satisfied.

MATLAB is used to calculate the equivalent resistant of each row of PV panels Rsim, based on Figure 6. For $5000 \mathrm{PV}$ panels, i.e. 1250 footing posts, Rsim=200.3 $\mathrm{m} \Omega$. The theoretical value of the equivalent resistant of each row is calculated by (4) to be Rinf. $=199.5 \mathrm{~m} \Omega$. Under a $220 \mathrm{kV}$ substation fault, the ground current is split into 113 rows, with an input impedance of $1.8 \mathrm{~m} \Omega$. The split factor is calculated to be 0.405 . The ground fault current is $6.076 \mathrm{kA}$. The designer will complete the split analysis between the $220 \mathrm{kV}$ substation earth grid and the input impedances from the 113 rows (for the purpose of this study, the connection impedance between the earth grid of the $220 \mathrm{kV}$ and the $132 \mathrm{kV}$ transformer that connect the row is considered to be $0.01 \mathrm{ohms}$ ).

The simulation shows that the current into each row is 54A and the grid current into the $220 \mathrm{kV}$ earth grid is $15 \mathrm{~A}$. The simulation shows that the maximum EPR for each row is $13 \mathrm{~V}$. The paper uses ETAP to verify the earth grid resistance of the rows and the maximum EPR under the fault conditions. ETAP software was used to compute the input resistance of each row. The results show an input resistance of $314 \mathrm{~m} \Omega$. It is worth noting that the authors ran an extreme case on ETAP where 5kA fault current was injected into one Row. The simulation shows that the maximum touch voltage is $158 \mathrm{~V}$ with an allowable touch voltage of $188 \mathrm{~V}$. Based on the case study data, there is no need for additional earth grid to ensure system compliance under given data. The system utilizes the existing foundations of the PV structures. This reduces the cost invested into the earthing system. Note that adequate bonding between the structures and the foundations has to be ensured, which is also required if electrodes and mesh are installed. For lightning assessment, the system has to comply with the surge impedance and surge voltages. Therefore, a designated electrode for the lightning masts might be required to absorb the lightning energy.

\section{CASE STUDY DISCUSSION}

Using the current available IEEE 80 standard [5], this paper proposed a case study to demonstrate the viability of the proposed design diagram. For the case study, the split factor and the substation EPR were calculated using the current IEEE design diagram; however, the designer did not take into consideration 
the connected PV rows. As shown in the results, the EPR is computed to be $2586 \mathrm{~V}$ due to a split factor of 0.193 . To validate the proposed design diagram, the case study is evaluated using the proposed steps shown in Figure 10. The EPR at the substation is computed to be $13.35 \mathrm{~V}$. Following the proposed diagram ensure that the design considers the foundation of the PV supports, which reduces the substation grid as seen from the fault. The results confirm the reduction in EPR. In addition, ETAP simulation shows that the system will comply with safety regulations if up to 5000 A of fault current reaches any row within the PV farm. Thus, this case validates the efficacy of the proposed design diagram.

\section{CONCLUSION}

The paper addresses an important topic related to safety and operation of PV solar farm. The work in this paper highlights the minimum requirements for the earthing system to meet safety and operational requirements with minimal costs. The presented design diagram offers the basic guidelines to ensure the system complies with the project requirements. The presented case studies show the advancement of the proposed diagram especially when it comes to cost reduction and man power deployments for the earthing system.

\section{REFERENCES}

[1] Solar PV power plant requires 40 miles of cable tray, Cabling Installation \& Maintenance, Accessed 13 June 2019. [Online]. Available: https://www.cablinginstall.com/articles/2016/01/fluor-solar-pv-cabletray.html

[2] "IEEE Guide for Measuring Earth Resistivity, Ground Impedance, and Earth Surface Potentials of a Grounding System," in IEEE Std 81-2012 (Revision of IEEE Std 81-1983), pp.1-86, 28 Dec. 2012.

[3] Australian/New Zealand Standard ${ }^{\mathrm{TM}}$ Earth potential rise-protection of telecommunication network users personnel and plant, Part 1: Code of practice, AS/NZS Std. 3835.1 2006, 27 September 2006.

[4] Australian/New Zealand Standard ${ }^{\mathrm{TM}}$ Earth potential rise-protection of telecommunication network users personnel and plant, Part 2: Application guide, AS/NZS Std. 3835.2 2006, 27 September 2006.

[5] IEEE Guide for Safety in AC Substation Grounding," in IEEE Std 80-2013 (Revision of IEEE Std 80-2000/ Incorporates IEEE Std 80-2013/Cor 1-2015), pp.1-226, 15 May 2015.

[6] Australian/New Zealand Standard ${ }^{\mathrm{TM}}$ Overhead line design-Detailed procedures, AZ/NZS 7000:2010, 26 November 2010.

[7] Australian/New Zealand Standard ${ }^{\mathrm{TM}}$ Electrical installations-surface mines and associated processing plant, AS/NZS Std. 3007.2-2004, 30 August 2004

[8] Nassereddine M, Rizk J, Nagrial M and Hellany A., "Estimation of apparent soil resistivity for two layers soil structure using the reflection coefficient K," International Journal of Energy and Environment and Economics, Vol.4, No. 4, pp. 573-580, 2013.

[9] Nassereddine M., Rizk J., and Nasserdine G., "Soil Resistivity Structure and Its Implication on the Pole Grid Resistance for Transmission Lines," International Journal of Electrical, robotics, electronics and Communications Engineering, Vol. 7, No. 1, pp. 19-23. 2013.

[10] Chow, Y L. Elsherbiny, M. and Salama M., "Resistance formulas for grounding systems in two-layer earth," IEEE Transactions on Power Delivery, Vol. 11, No. 3, pp. 1330-1336, 1996.

[11] Tsiamitros A., "Homogenous earth approximation of two-layer earth structures: an equivalent resistivity approach," IEEE Transactions on Power Delivery, Vol. 22, No. 1, pp. 658-666, 2006.

[12] W. Bogajewski, F. Dawalibi, Y. Gervais, D. Mukhedkar., "Effects of Sustained Ground Fault Current on Concrete poles," IEEE Power Engineering Review, Vol. PER-2, No. 8, pp. 43-43, 1982.

[13] Verma, R., D. Mukhedkar, "Ground fault current distribution in substation, tower and ground wire," IEEE transactions on power apparatus and systems, Vol. PAS-98, No. 3, 724-730, 1979.

[14] Nassereddine M., Hellany A., and Rizk J., "How to design an effective earthing system to ensure the safety of the people," International Conference on Advances in Computational Tools for Engineering Applications, pp. 416-421, 2009.

[15] [Online]. Available: http://grenlec.com/Blog/TabId/126/ArtMID/657/ArticleID/77/Solar-PV-Farm-Commissionedin-Carriacou.asp, Accessed on June 13, 2019.

[16] Nassereddine M., Rizk J., Hellany A. and Nagrial M., "Relation between transmission lines coupling factor and OHEW length; its impacts on fault current distribution," IET Generation, Transmission \& Distribution, Vol. 8, No. 4, pp. 600-608, 2014.

[17] Nassereddine M., Rizk J., Hellany A. and Nagrial M., "Transmission Mains pole earth potential rise (EPR) under substation fault," International journal of emerging electric power systems, Vol. 14, No. 5, pp. 449-507, 2013.

[18] C.N. Chang, "Computation of current-division factors and assessment of earth-grid safety at 161/69kV indoor-type and outdoor-type substations," IEE proc.-Gener. Transm. Distrib., Vol. 152, No. 6, pp. 837-848, 2005.

[19] Sullivan J. A., "Alternative earthing calculations for grid and rods," IEE proc. Gener. Transm. Distrib., Vol. 145, No. 3, pp. 271-280, 1998

[20] Vintan M. and Buta A., "Ground fault current distribution on overhead Tra20nsmission Lines," SER. Elec. Energ., Vol. 19, pp. 71-84, 2006. 
[21] Nassereddine M., Rizk J., Nagrial M. and Hellany A. "Transmission mains earthing design and concrete pole deployments," World Academy of Science, Engineering and Technology, Vol. 6, Issue 12, pp. 1681-1686, 2012

[22] Hans R., Arora J. and Soni S., "A practical approach for computation of grid current," IEEE Transactions on Power Delivery, Vol. 14, Issue 3, pp. 897-902, 1999

[23] Seppanen M, Tammi, O, Haarla C., "Underground Ground Wires for Transmission Lines: Electrical Behavior and Feasibility," IEEE Trans Power Delivery, Vol. 28, Issue 1, pp. 206-215, 2013.

[24] Uyttersprot L., Roca J., Chano S., Dormes L., Dos A., Kemmlert J., "Electrical Charateristics of Mixed Overhead Line/Cable Circuits," CIGRE Study Committee B5 Colloquium, Lausanne Switzerland, September 12-17, 2011.

[25] Cunnie E., "Ground Testing of Transmission Towers," IEEE $12^{\text {th }}$ International Conference on Transmission and Distribution, Operation and Live-Line Maintenance, pp. 1-8, 2011.

\section{BIOGRAPHIES OF AUTHORS}

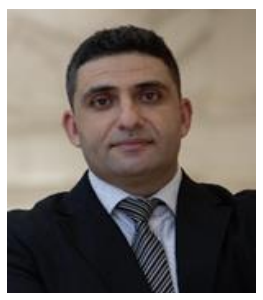

Dr. Mohamad Nassereddine, Assistant professor of electrical engineering, received his Ph.D. degree in the field of High Voltage Power Systems from Western Sydney University. He also received his MEng (Hons) by research in the field of Electric Machine and Renewable Energy from the same university. Dr. Nassereddine joined the American University in Dubai (AUD) in Fall 2017. Prior to joining AUD, he spent the past 13 years working in the academia and industry across Australia. Dr Nassereddine published large number of peer reviewed conferences and journals in the field of power system and renewable energy.

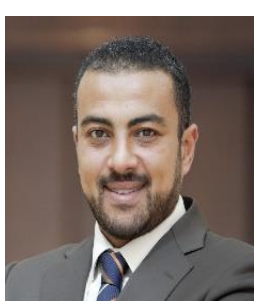

Dr. Khaled Ali obtained his Ph.D. in electrical engineering from University of Calgary, Canada. He joined the American University in Dubai (AUD) in Fall 2016 as an assistant professor of electrical engineering. Prior to joining AUD, Dr. Ali was a research fellow at Nanyang Technological University in Singapore, where he worked on enhancing vehicle localization in GNSS-denied environment. Prior that, Dr Ali's research was on characterization and mitigation of interference between digital subscriber line and power line communication networks.

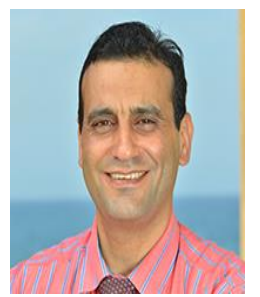

Dr. Chadi Nohra, Associate Professor for Electrical Power and Machines Engineering program at Beirut Arab University, received his PHD degree in the field of Control and Automation from Paul Cezanne University - Aix Marseille III- France. Dr. Nohra Joined Beirut Arab University in Fall 2012. His current research interests include electrical machines control and diagnostics, Diesel modeling \& diagnostics, renewable energy and advance power system for embedded generation and electric vehicles. 